\title{
Side population cells and drug resistance in breast cancer
}

\author{
MEIJIAN WANG*, YAQI WANG* ${ }^{*}$ and JUN ZHONG \\ Department of Oncology, Jiangxi Provincial Tumor Hospital, Nanchang, Jiangxi 330029, P.R. China
}

Received January 7, 2014; Accepted October 31, 2014

DOI: $10.3892 / \mathrm{mmr} .2015 .3291$

\begin{abstract}
Several studies have demonstrated that the isolated side population (SP) cells from solid tumors exhibit cancer stem cell-like properties, and are responsible for drug resistance during chemotherapy and tumor recurrence. In the current study, cancer stem cell-like SP cells were isolated in the MDU-22 breast cancer cell line using the Hoechst-33342 dye exclusion technique. It was observed that SP cells accounted for $3.8 \%$ of cells in the MDU-22 cell line, which was reduced to $0.6 \%$ in the presence of verapamil, an inhibitor of the ABC transporter. The sorted SP cells showed an elevated expression of stem cell markers, including $A B C G 2, O C T-4$ and EpCAM. Furthermore, it was demonstrated that the isolated SP cells undergo rapid proliferation and have a high survival rate. These results indicate that the coexpression of adenosine triphosphatase binding cassette transporters and stem cell surface markers in SP cells may contribute to chemoresistance, tumor recurrence, metastasis and invasion. Therefore, the isolation and characterization of SP cells may provide novel insights for the development of alternative therapeutic agents to target cancer stem cells.
\end{abstract}

\section{Introduction}

Breast cancer is one of the most common types of cancer, accounting for $13.7 \%$ of cancer-related mortalities in 2008 according to the World Cancer Research cancer report (1). Several factors have a key role in breast carcinogenesis, including ovarian steroids, estrogen and progesterone. Breast tumors are usually screened for hormone receptors, for example estrogen receptor (ER), progesterone receptor (PR) and overexpression of HER2, and based on the expression levels, patients are directed to endocrine or chemotherapy. It

Correspondence to: Dr Jun Zhong, Department of Oncology, Jiangxi Provincial Tumor Hospital, 519 Beijing East Road, Nanchang, Jiangxi 330029, P.R. China

E-mail:wanli0222@gmail.com

${ }^{*}$ Contributed equally

Key words: cancer stem cells, side population, transporters, multi-drug resistance, chemotherapy has been determined that the failure of cancer treatment is due to the persistence of cancer stem cells (CSCs) that evade the treatment regimen and are responsible for minimal residual disease (2). These cells have been found to possess characteristics usually associated with stem cells, such as self-renewal, and exhibit a high in vivo tumorigenicity, differentiation potential multi-drug and apoptosis resistance (3). Cells that exclude Hoechst 33342 dye are referred to as side population (SP) cells. These cells share characteristics with CSCs; specifically they are enriched for tumor initiating capacity, express stem-like genes and are resistant to chemotherapeutic drugs. Furthermore, SP cells also overexpress the adenosine triphosphatase binding cassette (ABC) transporters ABCB1 [multi-drug resistance transporter 1 (MDR1)], ABCC1 and ABCG2 (BCRP1), which contribute to multi-drug resistance and express stem cell surface markers. SP cells have been identified in several solid tumors and cancer cell lines based on Hoechst 33342 dye efflux by fluorescence activated cell sorting (FACS) (4-6). SP cells have been isolated from malignant and non-malignant tissues and they have been shown to exhibit stem cell characteristics and drug resistance (7-9). It has been reported that the presence of SP cells in mammary gland tissue of breast cancer accounts for 2.0-3.0\% of total epithelial cells in mice (8) and 2.0-5.0\% in humans (10). These SP cells have overexpression of $\mathrm{ABC}$ transporters, including ABCG2 and ABCB1 (MDR1) (11), and a high expression level of stem cell surface markers, which contribute to multi-drug resistance, high proliferation and survival rate, respectively (7). Hence, the sorting and characterization of SP cells would assist in elucidating the mechanisms of oncogenesis and drug resistance (8) and aid in the design of novel therapeutic strategies that may selectively target CSCs. Consequently, the present study is designed to isolate SP cells in the MDU-22 breast cancer cell line and analyze their expression levels of $\mathrm{ABC}$ transporters and stem cell surface markers.

\section{Materials and methods}

Cell line and cell culture. Samples from breast cancer tissues were obtained from a range of patients at the time of surgery. Written informed consent was obtained from all participants. All the patients were recruited from the Jiangxi Provincial Tumor Hospital (Jiangxi, China). Ethical approval was obtained from the Jiangxi Provincial Tumor Hospital. The patients details were as follows: Age range, 33-43 years; region, ductal mammary gland; grade, 4 (recurrent type). The tumor samples were washed immediately and tissues were 
broken down using collagenase (Sigma-Aldrich, Shanghai, China). The cells were continuously cultured in Dulbecco's modified Eagle's medium (DMEM; Sigma-Aldrich) and a cell line, MDU-22, was established. The cell line was cultured in DMEM with $10 \%$ fetal bovine serum (Life Technologies, Shanghai, China), supplemented with antibiotics (penicillin and streptomycin; Sigma-Aldrich) and maintained in T-75 flasks at $37^{\circ} \mathrm{C}$ in a humidified $5 \% \mathrm{CO}_{2}$ and $95 \%$ air atmosphere. Once confluent, cells were removed from the culture flask using Trypsin-EDTA $(0.25 \%, 53 \mathrm{mM}$ EDTA) washed, cells suspended in 10\% DMEM and centrifuged at 6,200 $\mathrm{x} g$ for 6 min. Cells were resuspended in 10\% DMEM. Cell count was measured using a hemocytometer (Bio-Rad, Inc., Shanghai, China).

Labeling with Hoechst 33342. All reagents mentioned in this paragraph were obtained from Sigma-Aldrich, with the exception of those already specified. The cells were divided into two groups, control cells + Hoechst $33342(n=4)$ and verapamil treated cells + verapamil + Hoechst $33342(n=4)$. Cells in staining medium $\left(\sim 10^{6}\right.$ cells $/ \mathrm{ml}$ of $10 \%$ DMEM) were labeled with Hoechst 33342 stock (Sigma-Aldrich) and bis-benzimide $(5 \mu \mathrm{l} / \mathrm{ml})$, either with dye alone or in combination with verapamil $(0.8 \mu \mathrm{l} / \mathrm{ml})$. The cells were mixed and placed in water bath at $37^{\circ} \mathrm{C}$ for exactly $90 \mathrm{~min}$. Subsequently, the cells were spun down $\left(2,000 \mathrm{rpm}\right.$ for $10 \mathrm{~min}$ at $\left.4^{\circ} \mathrm{C}\right)$ and resuspended in $500 \mu \mathrm{l}$ of Hank's buffered salt solution containing $10 \mathrm{mM}$ 4-(2-hydroxyethyl)-1-piperazineethanesulfonic acid. Finally, the cells were counter stained with propidium iodide (PI; $2 \mu \mathrm{g} / \mathrm{ml}$ sample) at $4^{\circ} \mathrm{C}$. The cells were filtered through a $50 \mu \mathrm{m}$ nylon mesh (BD Biosciences, Franklin Lakes, NJ, USA) into labeled FACS tubes in order to remove cell clumps. Separate tubes with fresh medium (10\% DMEM) were kept for sterile sorting of SP cells and main population cells. The cells were sorted using a FACSAria II flow cytometer (BD Biosciences, Mountain View, CA, USA). A wavelength of $355 \mathrm{~nm}$ was used to excite the Hoechst 33342 dye, and its dual-wavelength fluorescence was analyzed (blue, $450 \mathrm{~nm}$; red, $675 \mathrm{~nm}$ ).

In vitro proliferation activity assay. The sorted SP and non-SP cells were seeded into a 96 -well plate at a density

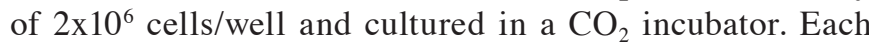
group was set up in triplicate. Cell proliferation activity was measured every day for 7 days. Each well was supplemented with CCK-8 solution $(10 \mu \mathrm{l})$ and incubated in $\mathrm{CO}_{2}$ incubator for 2-3 h. The optical density (OD) was determined at a wavelength of $450 \mathrm{~nm}$. These data were used to calculate cell growth graphs based on the mean value of $\mathrm{OD}_{450}$ and standard deviation values for each well.

Cell resistance assay. The obtained SP and non-SP cells were cultured in 96-well plates at a concentration of $1 \times 10^{3}$ cells/plate. After 24 h, 5-fluorouracil (5-FU) was added to all cultures to a final concentration of $10 \mu \mathrm{g} / \mathrm{ml}$. The plates were placed in a hatch box for $48 \mathrm{~h}$. Each well was supplemented with CCK-8 (10 $\mu \mathrm{l})$ solution and the plates were incubated for $3 \mathrm{~h}$. The mean value of $\mathrm{OD}_{450}$ obtained was represented as a graph. Cell resistance in the two groups was calculated using the following formula: Cell resistance $(\%)=\left(\right.$ experimental group $\mathrm{OD}_{450}$ value/control group $\mathrm{OD}_{450}$ value) x 100 .
Immunocytochemistry. The sorted SP cells and non-SP cells were seeded into $35 \mathrm{~mm}$ culture plates $(\sim 100 \mu \mathrm{l})$, maintained in an incubator for $3 \mathrm{~h}$ and supplemented with $1 \mathrm{ml} \mathrm{DMEM} \mathrm{(10 \% ).}$ Following overnight incubation, the cells were rinsed with phosphate-buffered saline (PBS; Life Technologies) and fixed in $4 \%$ paraformaldehyde (Life Technologies) in $1 \mathrm{X}$ PBS, for $5 \mathrm{~min}$ at $4{ }^{\circ} \mathrm{C}$. After washing with $1 \mathrm{X}$ PBS, cells were blocked with $1 \%$ bovine serum albumin in Tris-buffered saline (BSA-TBS; Life Technologies) with RNase (10 $\mu 1$ per $1,000 \mu \mathrm{l}$ of $3 \%$ BSA-TBS; Life Technologies). Following a 1 -h incubation at room temperature the cells were rinsed with PBS, and FAK primary mouse polyclonal antibody (Life Technologies) in 1\% BSA-TBS was added (dilution, $1: 100 ; 2 / 200 \mu \mathrm{l}$ ) prior to incubation overnight at $4^{\circ} \mathrm{C}$. Once washed with $1 \mathrm{X}$ PBS, the cells were incubated with Rabbit Anti-Mouse $\operatorname{IgG}(\mathrm{H}+\mathrm{L})$ Superclonal ${ }^{\mathrm{TM}}$ Secondary antibody (dilution, 1:100 in 1\% BSA-TBS), at room temperature for $1 \mathrm{~h}$. The cells underwent a further PBS wash and PI was added $(1 / 200 \mu 1$ of PBS). The cells were viewed under a confocal laser scanning microscope (Leica TCS; Leica Microsystems, Wetzlar, Germany). Image analysis and figures were prepared using Adobe Photoshop CS4 (Adobe Systems, Inc., San Jose, CA, USA).

Reverse transcription quantitative polymerase chain reaction $(R T-q P C R)$. PCR was carried out as previously described (13). Total RNA was isolated from the SP and non-SP cells using the Ambion RNAqueous ${ }^{\circledR}$ Micro kit (Applied Biosystems, Warrington, UK). cDNA was synthesized using the Bioline cDNA synthesis kit (Bioline, London, UK). qPCR was performed using 2-3 $\mu$ l cDNA and 2X TaqMan Gene Expression Master mix (Applied Biosystems) in $30 \mu \mathrm{l}$ reaction volumes. GAPDH was used as a reference. The primer sequences were as follows (14): forward, 5'-AGCTGCAAGGAAAGATCCAA-3' and reverse, 5'-TCCAGACACACCACGGATAA-3' for ABCG2; forward, 5'-ATCCTGGGGGTTCTATTTGG-3' and reverse, 5'-CTCCAGGTTGCCTCTCACTC-3' for OCT-4; forward, 5'-CTGCCAAATGTTTGGTGATG-3' and reverse, 5'-ACG CGTTGTGATCTCCTTCT-3', for epithelial cell adhesion molecule (EpCAM); and forward, 5'-ATGTCGTGG AGTCTACTGGC-3' and reverse, 5'-TGACCTTGCCCA CAGCCTTG-3' for GAPDH. The amplified products were separated by electrophoresis on ethidium bromide-stained $1.2 \%$ agarose gels. Band intensity was measured by ImageJ (National Institutes of Health, Bethesda, MD, USA) from two independent experiments.

Statistical analysis. A one-way analysis of variance test was performed to determine any differences between the treatment groups. Student's t-tests were performed to compare the effect of different treatments between the SP and non-SP populations. ${ }^{*} \mathrm{P}<0.05$ and ${ }^{* *} \mathrm{P}<0.01$ were considered to indicate a statistically significant difference.

\section{Results}

Analysis of SP Cells FACS using Hoechst 33342. The live cell population (P1 gated region) was selected against PI as the P1 gated population. PI is used to exclude the dead 


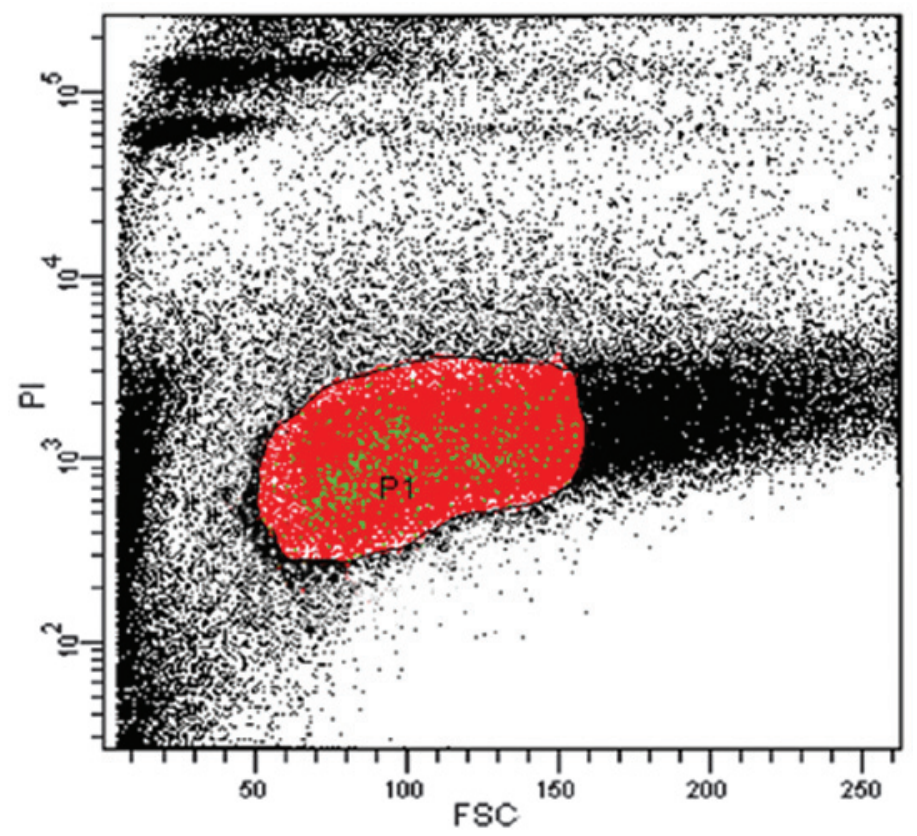

Figure 1. A dot blot analysis by fluorescence activated cell sorter (FACS) clearly showing the live cells in the P1 gated population. Cells were stained with propidium iodide (PI) to exclude the dead cells.

A

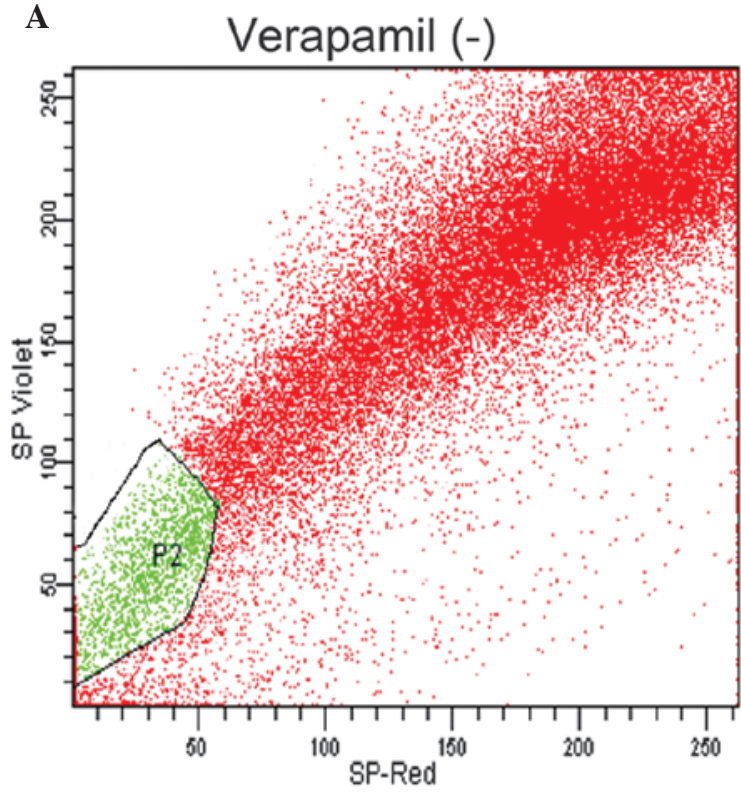

B

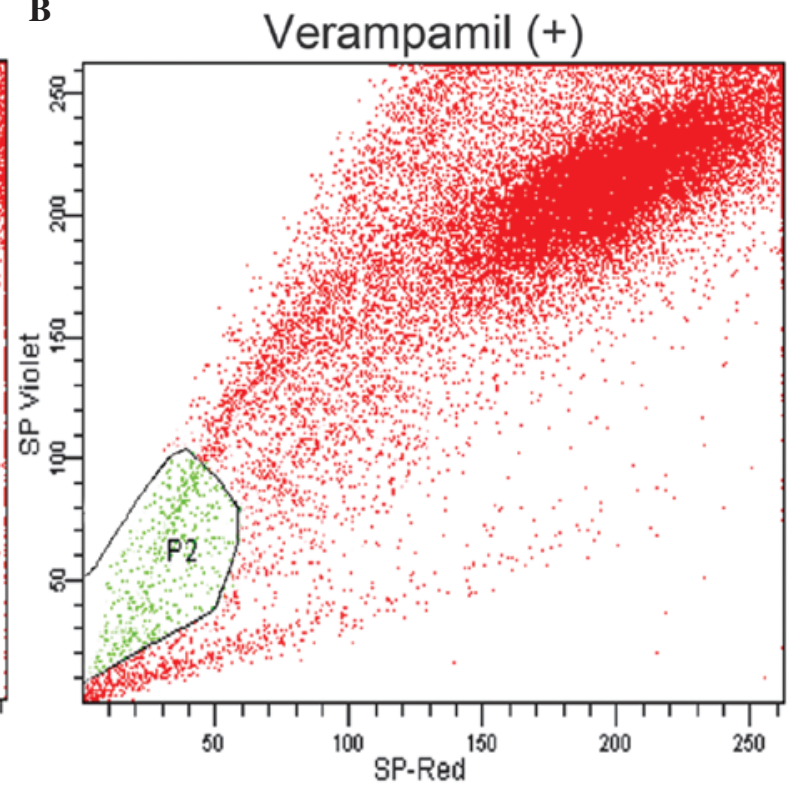

Figure 2. Analysis of side population (SP) cells in the breast cancer cell line. (A) MDU-22 cells were stained using Hoechst 33342 dye and analyzed using flow cytometry. SP cells are outlined (P2-gated population) and also shown as a percentage (3.8) of the total cell population. (B) Percentage of SP cells was significantly reduced $(0.6)$ in the presence of verapamil.

cells from the sample (Fig. 1). SP cells were sorted out from the gated live cell population (P1) using Hoechst 33342 , which is a DNA binding dye. Hoechst 33342 emits light at wavelengths of $\sim 450 \mathrm{~nm}$ (SP-Violet) and $675 \mathrm{~nm}$ (SP-Red) following UV excitation, and a set of cells were observed that displayed low blue and red fluorescence. This distinct cell population (P2) found towards the SP-violet region of the dot plot of the FACS profile are the so-called SP cells (Fig. 2A). The exclusion of Hoechst 33342 by SP cells is an active process involving MDR1, a member of the ABC transporter transmembrane proteins (Fig. 2A). The number of cells collected was $\sim 62 \times 10^{3}$, which is $\sim 56 \%$ of the initial cell count. Of the $62 \times 10^{3}$ cells (P1) analyzed, Hoechst dye was effluxed by $3.8 \%$ of cells in the $\mathrm{P} 2$ gated region (Fig. 2A). Verapamil-treated cells of the same cell line were sorted out with the same Hoechst 33342 efflux. The resulting P1 cells obtained were $\sim 48,800$ in number, which was $\sim 44 \%$ of the initial cell count. Following treatment with verapamil, the percentage of SP cells (P2 gated) was reduced from 3.8 to $0.6 \%$ (Fig 2B). Verapamil is a MDR1 transporter protein inhibitor, which blocks the drug efflux action by the cells. Hence, the sorted SP cells are highly resistant to drug uptake, 
A

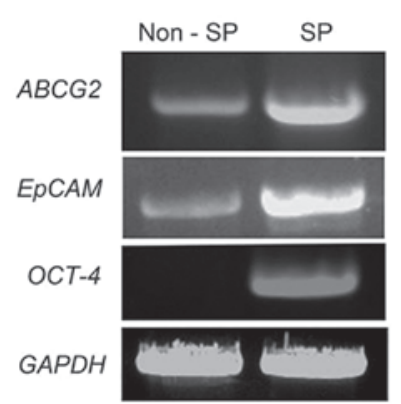

B

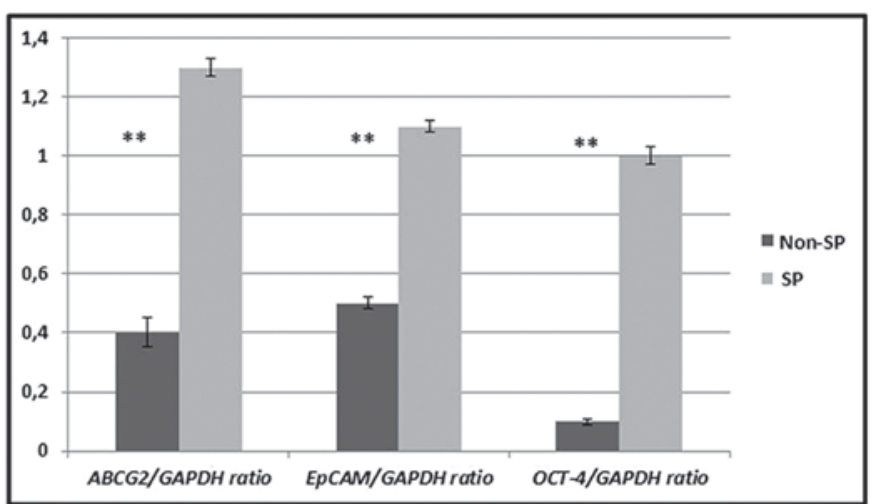

Figure 3. Expression of stem cell markers in side population (SP) and non-SP cells. (A) Elevate expressions of ABCG2, OCT-4 and EpCAM genes in derived MDU-22 were detected by reverse transcription-quantitative polymerase chain reaction. (B) Quantification graph represents the data of two separate independent experiments. The bars represent standard deviation. ${ }^{* *} \mathrm{P}<0.01$ compared with parental and non-SP cells, respectively.

Non-SP cells

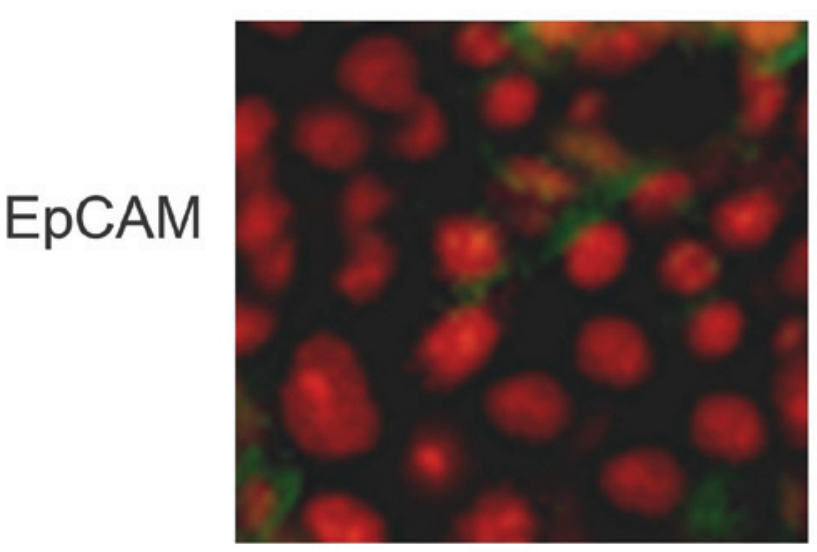

SP cells

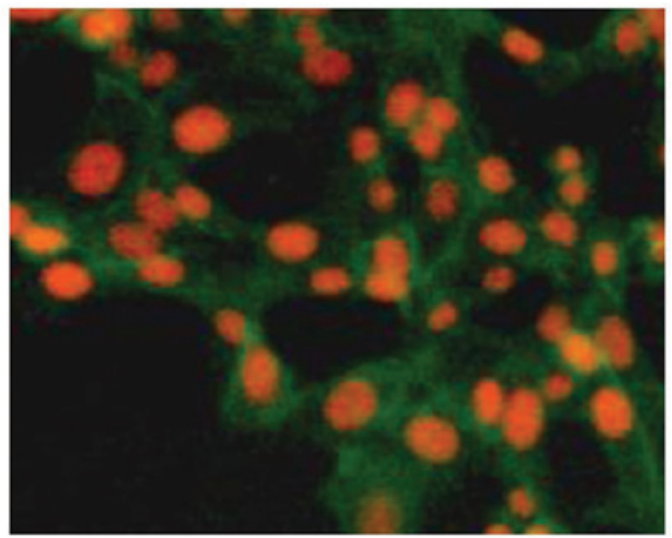

Figure 4. Fluorescence microscopic analysis of cell surface markers. The fluorescence intensity of EpCAM is highly enriched in side population (SP) cells whereas this fluorescence intensity is reduced in non-SP.

A

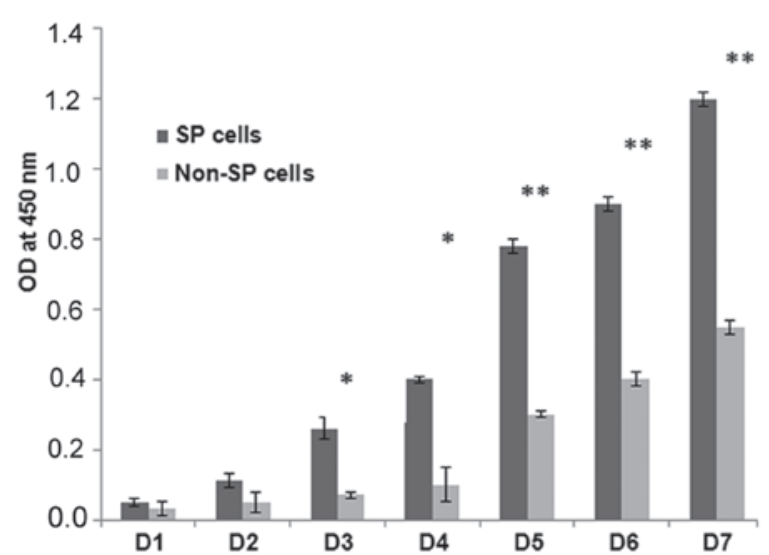

B

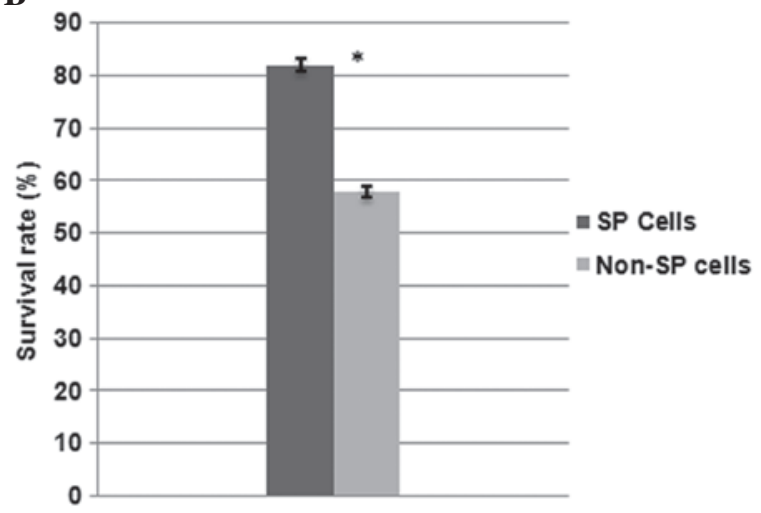

Figure 5. (A) Cell proliferation rate for side population (SP) and non-SP cells. The $\mathrm{x}$-axis represents time, while the y-axis indicates the corresponding optical density (OD) value at $450 \mathrm{~nm}$ with ${ }^{*} \mathrm{P}<0.05{ }^{*}{ }^{*} \mathrm{P}<0.01$. (B) Resistance of SP cells and non-SP cells to 5 -fluorouracil (5-FU). The y-axis shows the survival rate of the two groups following treatment with $10 \mu \mathrm{g} / \mathrm{ml} 5-\mathrm{FU}$ for $3 \mathrm{~h}$.

which may be due to the over expression of $\mathrm{ABC}$ transporters. Therefore, these cells were further analyzed for the expression of $A B C$ transporters and stem cell surface markers.
Analysis of ABCG2 expression and stem cell surface markers in SP cells. It has been previously reported that ABCG2 is expressed by malignant breast tissue, and it was observed that 
ABCG2 expression increases with higher tumor grades $(15,6)$. Therefore, to examine and compare the expression level of the $A B C B 2$ gene and other stem cell genes, including OCT-4 and EpCAM, between SP and non-SP cells, the extracted RNA was analyzed using RT-qPCR. The $A B C B 2, E p C A M$ and $O C T-4$ levels were higher in SP cells compared to non-SP cells (Fig. 3A). The quantification graph clearly shows that the levels of these genes in SP cells are significantly higher (Fig. 3B). GAPDH was used as a reference gene. Notably, fluorescence microscopic analysis revealed that, compared with the non-SP cells, the SP cells displayed a greater positive expression of cell surface proteins such as EpCAM (Fig. 4) which further confirms the RT-qPCR results. These results suggest that a high expression level of $A B C G 2$ and $O C T-4$ in SP cells may act as a crucial factor in drug resistance and the massive proliferation of cancer cells.

Characterization of sorted SP cells. FACS sorted SP and non-SP cells were further subjected to in vitro cell proliferation and cell survival assays. The isolated breast carcinoma SP cells underwent rapid cell proliferation starting from the third day and becoming more confluent on the eighth day (data not shown) (Fig. 5A). However, the growth rates of the non-SP cells were significantly lower when compared with those of the SP cells (Fig. 5A). The measured growth rate of SP cells at $450 \mathrm{~nm}$ was significantly higher than non-SP cells $\left({ }^{*} \mathrm{P}<0.05 ;{ }^{* *} \mathrm{P}<0.01\right)$. Following the in vitro proliferation assay, the SP cells were further subjected to a drug resistance assay. The survival rate of the SP cells (83\%) following exposure to $10 \mu \mathrm{g} / \mathrm{ml} 5$-FU were significantly higher (Fig. 5B) compared with that of the non-SP cells (58\%). Hence these data suggest that the sorted SP cells have high proliferation rate and an increased resistance to chemotherapeutic agent.

\section{Discussion}

CSCs have the ability to resist chemotherapeutic agents via increased expression levels of efflux pumps, and they have an enhanced DNA repair activity. The theory of cancer stem cells hypothesizes that treatment failure and minimal residual disease are caused by the presence of small population of CSCs $(7,8)$, which are responsible for tumor growth, metastasis and tumor relapse. Hence the eradication of CSCs is an important goal for providing effective cancer treatment and long term disease-free survival.

SP cells have been observed in several types of solid tumors as well as a number of breast cancer cell lines $(7,9)$. SP cells were first identified in primary human breast cancer cells, and they demonstrated the expression of HER2 signaling which is involved in drug efflux via $A B C$ transporters (16). In the present study, we demonstrated that SP cells was identified and isolated from breast carcinoma cell line (MDU-22). SP cells were sorted out based on Hoechst 33342 dye exclusion by FACS. The exclusion of Hoechst 33342 by SP cells is due to highly induced ABCG2 (MDR1), a member of the ABC transporter transmembrane proteins that are involved in multi-drug resistance (Fig. 2A). The results of the current study demonstrated that, following treatment with verapamil (a MDR1 protein inhibitor), the percentage of SP cells was reduced from 3.8 to $0.6 \%$
(Fig. 2B). This is a confirmatory test for the presence of MDR1 protein in MDU-22 cells. Furthermore, the results of the RT-qPCR showed that the expression levels of genes such as $A B C G 2, O C T-4$ and EpCAM are significantly higher in SP cells than those in the non-SP population (Fig. 3). A previous study has shown that the ABCG2 protein is over expressed in the MCF-7 cell line, and that these cells were more drug resistant compared with the MCF-7 non-SP cells (6). Therefore, the results of the current study fit well with the recent study by Britton et al (6), 2012 and with other previous studies $(9,17)$ where a differential increase in the expression levels of mRNA encoding $A B C G 2$ was observed in the SP population when compared with that of the non-SP cells. Similarly, the OCT-4 gene, a member of the POU family of transcription factors, was shown to be involved in the proliferation potential (18) and survival of CSCs partly through the OCT-4/Tcl1/Akt1 pathway (19). Hence the results of the current study indicate that the overexpression of ABCG2 may act as a suitable marker for the identification of SP cells in breast cancers. Subsequently, the results revealed that SP cells displayed a greater positive expression of cell surface markers such as epithelial cell adhesion molecule (EpCAM), a CSC marker (Fig. 4). In line with the present results, CD44 cells in the head and neck squamous cell carcinomas of mice were proven to be enriched with tumorigenic CSCs able to propagate tumor formation, whereas CD44- cells were not able to form tumors (5).

Furthermore, the in vitro proliferation and drug resistance assays revealed that MDU-22 SP cells have an increased proliferation capacity and are highly resistant to 5-FU, hence they have increased survival rate compared with that of the non-SP cells, which are very sensitive (Fig. 4). It has been previously reported that MDA-MB-231 cell lines have a significantly increased survival rate when treated with doxorubicin, methotrexate and 5-FU, compared with untreated cells (20). Taken together, the results of the present study indicate that the sorted SP cells show elevated expression levels of $A B C G 2, O C T-4$ and $E p C A M$, hence, the interactions of these genes may be collectively involved in the drug resistance and enhanced survival rate of SP cells. However, the signaling pathways and cascade of events involved in expression of these genes in CSCs remains speculative. However, the identification and characterization of SP cells provides a strategy to design novel therapeutic targets with the eventual goal of eliminating residual disease and preventing tumor recurrence.

\section{References}

1. World Cancer Report. International Agency for Research on Cancer. 2008. Retrieved 2011-02-26. (cancer statistics often exclude non-melanoma skin cancers such as basal cell carcinoma which though very common are rarely fatal).

2. Ramachandran C and Melnick SJ: Multidrug resistance in human tumors - molecular diagnosis and clinical significance. Mol Diagn 4: 81-94, 1999.

3. Gil J, Stembalska A, Pesz KA and Sasiadek MM: Cancer stem cells: the theory and perspectives in cancer therapy. J Appl Genet 49: 193-199, 2008.

4. Robey RW, Shukla S, Finley EM, et al: Inhibition of P-glycoprotein (ABCB1)- and multidrug resistance-associated protein 1 (ABCC1)-mediated transport by the orally administered inhibitor, CBT-1((R)). Biochem Pharmacol 75: 1302-1312, 2008. 
5. Yanamoto S, Kawasaki G, Yoshitomi I, Iwamoto T, Hirata K and Mizuno A: Clinicopathologic significance of EpCAM expression in squamous cell carcinoma of the tongue and its possibility as a potential target for tongue cancer gene therapy. Oral Oncol 43: 869-877, 2007.

6. Britton KM, Eyre R, Harvey IJ et al: Breast cancer, side population cells and ABCG2 expression. Cancer Lett 323: 97-105, 2012.

7. Hirschmann-Jax C, Foster AE, Wulf GG, et al: A distinct 'side population' of cells with high drug efflux capacity in human tumor cells. Proc Natl Acad USA 101: 14228-14233, 2004.

8. Kondo T, Setoguchi T and Taga T: Persistence of a small subpopulation of cancer stem-like cells in the C6 glioma cell line. Proc Natl Acad Sci USA 101: 781-786, 2004.

9. Patrawala L, Calhoun T, Schneider-Broussard R, Zhou J, Claypool K and Tang DG: Side population is enriched in tumorigenic, stem-like cancer cells, whereas $\mathrm{ABCG}^{+}$and $\mathrm{ABCG}^{-}$cancer cells are similarly tumorigenic. Cancer Res 65: 6207-6219, 2005.

10. Welm BE, Tepera SB, Venezia T, et al: Sca-1(pos) cells in the mouse mammary gland represent an enriched progenitor cell population. Dev Biol 245: 42-56, 2002.

11. Clarke R, Spence K, Anderson E, Howell A, Okano H and Potten C: A putative human breast stem cell population is enriched for steroid receptor-positive cells. Dev Biol 277: 443-456, 2005.

12. Jonker JW, Freeman J, Bolscher E, et al: Contribution of the ABC transporters Bcrpl and Mdrla/lb to the side population phenotype in mammary gland and bone marrow of mice. Stem Cells 23: $1059-1065,2005$
13. Yanamoto S1, Kawasaki G, Yamada S, Yoshitomi I, et al: Isolation and characterization of cancer stem-like side population cells in human oral cancer cells. Oral Oncol 47: 855-860, 2011.

14. Britton KM1, Eyre R, Harvey IJ, Stemke-Hale K, et al: Breast cancer, side population cells and ABCG2 expression. Cancer Lett 323: 97-105, 2012.

15. Faneyte IF, Kristel PM, Maliepaard M, Scheffer GL, Scheper RJ, Schellens JH and van de Vijver MJ: Expression of the breast cancer resistance protein $\mathrm{n}$ breast cancer. Clin Cancer Res 8: 1068-1074, 2002.

16. Nakanishi T, Chumsri S, Khakpour N, et al: Side-population cells in luminal-type breast cancer have tumour-initiating cell properties, and are regulated by HER 2 expression and signaling. Br J Cancer 102: 815-826, 2010.

17. Engelmann K, Shen K and Finn OJ: MCF7 side population cells with characteristics of cancer stem/progenitor cells express the tumor antigen MUC1. Cancer Res 68: 2419-2426, 2008.

18. Campbell PA, Perez-Iratxeta C, Andrade-Navarro MA and Rudnicki MA: Oct4 targets regulatory nodes to modulate stem cell function. PLoS One 2: e553, 2007.

19. Hu T, Liu S, Breiter DR, Wang F, Tang Y and Sun S: Octamer 4 small interfering RNA results in cancer stem cell-like cell apoptosis. Cancer Res 68: 6533-6540, 2008.

20. Steiniger SC1, Coppinger JA, Krüger JA, Yates J III and Janda KD: Quantitative mass spectrometry identifies drug targets in cancer stem cell-containing side population. Stem Cells 26: 3037-3046, 2008. 